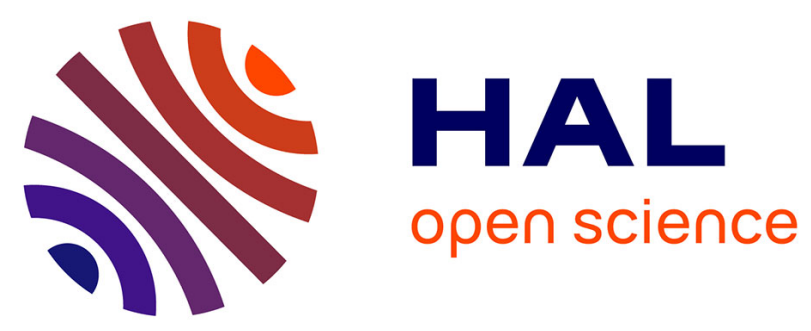

\title{
Adaptive Basis Scan by Wavelet Prediction for Single-pixel Imaging
}

Florian Rousset, Nicolas Ducros, Andrea Farina, Gianluca Valentini, Cosimo d'Andrea, Françoise Peyrin

\section{- To cite this version:}

Florian Rousset, Nicolas Ducros, Andrea Farina, Gianluca Valentini, Cosimo d'Andrea, et al.. Adaptive Basis Scan by Wavelet Prediction for Single-pixel Imaging. IEEE Transactions on Computational Imaging, 2017, 3 (1), pp.36-46. 10.1109/TCI.2016.2637079 . hal-01314314v2

\section{HAL Id: hal-01314314 \\ https://hal.science/hal-01314314v2}

Submitted on 12 Dec 2016

HAL is a multi-disciplinary open access archive for the deposit and dissemination of scientific research documents, whether they are published or not. The documents may come from teaching and research institutions in France or abroad, or from public or private research centers.
L'archive ouverte pluridisciplinaire HAL, est destinée au dépôt et à la diffusion de documents scientifiques de niveau recherche, publiés ou non, émanant des établissements d'enseignement et de recherche français ou étrangers, des laboratoires publics ou privés. 


\title{
Adaptive Basis Scan by Wavelet Prediction for Single-pixel Imaging
}

\author{
Florian Rousset, Nicolas Ducros, Andrea Farina, Gianluca Valentini, Cosimo D’Andrea and Françoise Peyrin
}

\begin{abstract}
Single pixel camera imaging is an emerging paradigm that allows high-quality images to be provided by a device only equipped with a single point detector. A single pixel camera is an experimental setup able to measure the inner product of the scene under view -the image- with any user-defined pattern. Post-processing a sequence of point measurements obtained with different patterns permits to recover spatial information, as it has been demonstrated by state-of-theart approaches belonging to the compressed sensing framework.

In this paper, a new framework for the choice of the patterns is proposed together with a simple and efficient image recovery scheme. Our goal is to overcome the computationally demanding $\ell_{1}$-minimization of compressed sensing. We propose to choose patterns among a wavelet basis in an adaptive fashion, which essentially relies onto the prediction of the significant wavelet coefficients' location.

More precisely, we adopt a multiresolution strategy that exploits the set of measurements acquired at coarse scales to predict the set of measurements to be performed at a finer scale. Prediction is based on a fast cubic interpolation in the image domain. A general formalism is given so that any kind of wavelets can be used, which enables one to adjust the wavelet to the type of images related to the desired application.

Both simulated and experimental results demonstrate the ability of our technique to reconstruct biomedical images with improved quality compared to CS-based recovery. Application to real-time fluorescence imaging of biological tissues could benefit from the proposed method.
\end{abstract}

Index Terms-Single-pixel camera, wavelets, compressive sensing, optical imaging, fluorescence imaging.

\section{INTRODUCTION}

$\mathrm{T}$ HE SINGLE-PIXEL CAMERA (SPC) architecture [1], [2] enables to build small, low-cost, and high-quality imaging devices. When compared to CCD or CMOS cameras, several

This work is funded by the Universite Franco-Italienne and was performed within the framework of the LABEX PRIMES (ANR-11-LABX-0063) of Université de Lyon, within the program "Investissements d'Avenir" (ANR11-IDEX-0007) operated by the French National Research Agency (ANR). It was also partially supported by Cariplo Foundation under Grant N. 20130615. LASERLAB-EUROPE (grant agreement no. 284464, ECs Seventh Framework Programme).

F. Rousset is with Univ Lyon, INSA Lyon, UCBL, CNRS 5220 INSERM U1206, CREATIS, 69621 Villeurbanne Cedex, France and the Dipartimento di Fisica of the Politecnico di Milano, 20133 Milano, Italy. Corresponding author: florian.rousset@creatis.insa-lyon.fr.

N. Ducros and F. Peyrin are with Univ Lyon, INSA Lyon, UCBL, CNRS 5220 INSERM U1206, CREATIS, 69621 Villeurbanne Cedex, France.

A. Farina is with the Consiglio Nazionale delle Ricerche, IFN, 20133 Milano, Italy

G. Valentini is with the Dipartimento di Fisica of the Politecnico di Milano, 20133 Milano, Italy.

C. D'Andrea is with the Dipartimento di Fisica of the Politecnico di Milano, with the Center for Nano Science and Technology@PoliMi, Istituto Italiano di Tecnologia and with the Consiglio Nazionale delle Ricerche, IFN, 20133 Milano, Italy. advantages stand out. First, single detectors can have a high efficiency and are therefore able to detect weak light intensity changes [3]. This can be very useful for medical applications where tissue absorption can be quite high [4]. Second, small storage memory is needed given that compression is performed at the hardware level. This is an important advantage for applications needing remote imaging (e.g. aerospace remote sensing) where the data rate for transmission would be low [5], [6]. Finally, an imaging device based on a single point sensor is usually cheaper than one based on a sensor array. This makes the SPC a perfect candidate for infrared imaging [7] where it would be costly to use a conventional imaging system operating at these wavelengths [8]. All the mentioned advantages can benefit to several imaging fields such as 3D imaging [9], [10], ghost imaging [11], multispectral or hyperspectral imaging [12]-[15], terahertz imaging [16], [17] or video acquisition [18], [19]. The SPC can also be seen as an excellent candidate for medical imaging applications. Coupling the unique detector with a time-correlated single photon counting board allows one to create a low-cost time-resolved imaging system [15] (e.g. fluorescence lifetime imaging [20]). It can also be used for microscopy [21], [22], imaging through scattering media [23], [24] or for diffuse optics (e.g. intraoperative or skin lesions detection [25]). Exploitation of several SPC images is of interest for diffuse optical tomography or fluorescence molecular tomography [26]-[29], with application to oximetry and molecular imaging.

The compressive sensing (CS) paradigm [30] has been widely applied to optical systems [31], [32]. In particular, since the pioneering work of Duarte and coauthors [1], [2], SPC has been mainly associated to the CS that provides an excellent theoretical framework for recovering an image from SPC measurements. Recently, CS-based SPC found various applications [5], [6], [9], [10], [12]-[19], [22]-[24], [33]. The computationally expensive image recovery based on $\ell_{1}$ minimization is a drawback that can restrict the applicability of the SPC, e.g., to real-time applications and/or application requiring high-resolution images.

A second kind of approach permits a straightforward recovery of the image that avoids the $\ell_{1}$-minimization. The acquisition consists in a basis scan (BS), i.e., the SPC progressively acquires the scene under view in a known basis [34][37] (e.g. Hadamard, Fourier or wavelet). The image recovery simply consists in inverse transforming the measured data. While BS-SPC offers fast image restoration, it suffers from long acquisition times and/or is restricted to the acquisition of low-resolution images since the number of measurements is given by the number of pixels of the desired image. 
In recent years, adaptive schemes for BS-SPC have emerged. Adaptive basis scan (ABS) lies on the predictions of the most significant basis functions for the particular scene under view. Prediction is generally performed progressively during the experiment, exploiting the previously acquired data. Wavelet basis are of particular interest since $i$ ) most images are known to have a sparse representation in such basis and ii) fast inverse wavelet transform algorithms are available to restore the image quickly [38], [39].

The ABS framework mainly relies on the prediction step. In [40], the authors consider Haar's wavelet and use a thresholding technique together with the Lipschitz exponent method [39] to decide the coefficients to acquire. A similar approach with the same wavelet is used in [41] where a more refined prediction strategy is proven to outperform Deutsch's method [40]. Both techniques also rely on the father-son relationship between wavelet coefficients over resolution scales [39]. The main disadvantage of the thresholding strategies is the fact that thresholds are image-dependent and need adjustments. In [42], the Haar wavelet is also used and the prediction step is based on the statistical modeling of images and thresholding. Hybrid methods have also been investigated. In [43], the authors combine CS and Deutsch's ABS technique [40] for ghost imaging and the same approach is proposed in [44] for hyperspectral imaging. In [45], the authors divide the image into patches and perform a BS acquisition with Hadamard functions [46] at different resolutions. The acquisition for a given patch is decided based on the presence of information in this region. Moreover, adaptivity in the context of CS have been studied by several authors. Some argued there is not much benefit [30] while others claimed that it can reduce the number of measurements [47] or improve the measurements accuracy [48], [49].

In this paper, we propose a complete framework for SPC image acquisition and restoration using a new ABS technique, which benefits of two main features. First, we present a threshold-free prediction strategy inspired by the non-linear wavelet approximation. This is based on our work presented in [50] where a different prediction strategy was employed. The second feature is the ability to handle any kind of wavelet for acquisition. While Haar's wavelet, which is well adapted to the SPC technology, has been widely used, we show that more sophisticated wavelets can provide an improved image quality. The Matlab implementation of our ABS-WP method is available online together with several data sets [51]. In Section II, we present the CS-based conventional approach for SPC. In Section III, we present our method that we refer to as Adaptive Basis Scan by Wavelet Prediction (ABS-WP). We recall the important facts about the wavelet decomposition before detailing our acquisition strategy. A method to use any kind of wavelet is also presented. Section IV presents the conditions in which the experiments were made and Section $\mathrm{V}$ reports the associated results on both simulated and experimental data. A comparison between ABS-WP and CS is also given, extending the results in [52]. We discuss the results in Section VI where it is given some insights about the system's possibilities. Finally, our conclusions are reported in Section VII.

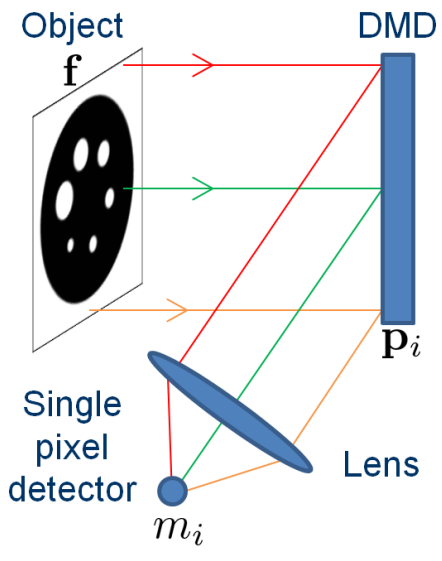

Fig. 1. Optical setup of the single-pixel camera using a DMD. The image is noted $\mathbf{f}, \mathbf{p}_{i}$ is a DMD pattern and $m_{i}$ is the corresponding measure.

\section{Conventional CS-BASED APPROACH}

A SPC consists of a spatial light modulator coupled with a single pixel detector. A common choice is the use of a digital micromirror device (DMD) as a spatial light modulator as illustrated in Fig. 1. A lens is added to focus light onto the single detector. A DMD has thousands of mirrors that can be independently tilted in two states. The ON state reflects the light toward the detector whereas the OFF state reflects the light in the opposite direction. Hence, a DMD can act as a tunable spatial filter, not only with black-and-white patterns but also with gray-level patterns. For this, the mirrors flip between the ON and OFF states in a predefined amount of time at a very high frequency. This enables contemporary DMD to produce up to 10-bits grayscales patterns.

\section{A. Single-pixel camera acquisition}

A SPC acquisition consists in experimentally measuring the inner product of an image and some DMD patterns, sequentially. Let $\mathbf{F} \in \mathbb{R}^{N \times N}$ be a $N \times N$ image whose units are in photons per second (ph/s) i.e. $\mathbf{F}$ is directly considered as the light source. We note $\mathbf{f} \in \mathbb{R}^{P \times 1}$ its vectorized form with $P=N^{2}$. The signal $m_{i}(\mathrm{ph})$ measured by the single detector during the integration time $\Delta t$ (s) may be modeled as

$$
m_{i}=\Delta t \mathbf{p}_{i}^{\top} \mathbf{f}
$$

where $\mathbf{p}_{i} \in \mathbb{R}^{P \times 1}$ (no unit) is a pattern loaded onto the DMD.

Let $\mathbf{P}=\left(\mathbf{p}_{1}, \ldots, \mathbf{p}_{I}\right)^{\top} \in \mathbb{R}^{I \times P}$ be the matrix containing the sequence of $I$ DMD patterns $\left\{\mathbf{p}_{i} \in \mathbb{R}^{P \times 1}, i=1 \ldots I\right\}$. The measurement vector $\mathbf{m}=\left(m_{1}, \ldots, m_{I}\right)^{\top} \in \mathbb{R}^{I \times 1}$ containing the sequence of measurements is given by the matrix equation

$$
\mathbf{m}=\Delta t \mathbf{P f}
$$

The previous equation suggests that implementing a SPC acquisition requires to solve the following two problems:

$\left(P_{1}\right)$ How to choose the set of DMD patterns $\mathbf{P}$ ?

$\left(P_{2}\right)$ How to restore the image $\mathbf{f}$ from the measurements $\mathbf{m}$ knowing the patterns $\mathbf{P}$ ? 


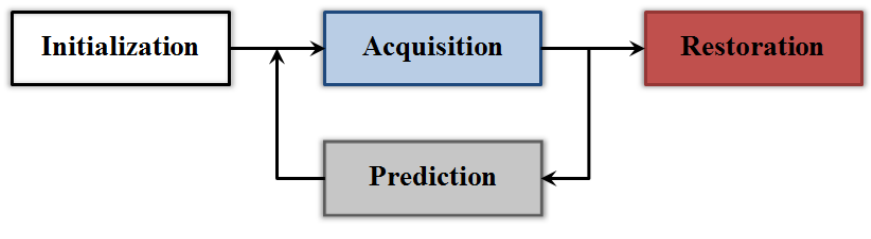

Fig. 2. Scheme of an adaptive acquisition framework for single-pixel camera.

\section{B. Compressive sensing acquisition and restoration}

The problem of the acquisition and recovery of a SPC image by means of CS was originally formulated in [1], [2]. The CS framework provides an elegant solution to problems $P_{1}$ and $P_{2}$ assuming that the image has a sparse representation in some basis $\boldsymbol{\Lambda}$. Mathematically,

$$
\mathbf{f}=\Lambda \mathbf{s}
$$

where $\mathbf{s} \in \mathbb{R}^{P \times 1}$ is $K$-sparse, i.e., only $K$ entries of $\mathbf{s}$ are nonzero. Typical choice for $\boldsymbol{\Lambda}$ includes wavelet basis, Fourier basis, and discrete cosine basis.

Solution to $P_{1}$ : The CS framework allows to consider only $I<<P$ measurements when the DMD patterns (the sensing matrix in the CS vocabulary) $\mathbf{P}$ is chosen as a random matrix satisfying the so-called restricted isometry property (RIP) [30], [53]. Henceforth, the entries of $\mathbf{P}$ are commonly chosen from independent and identically distributed realizations [2], [54] such as

$$
(\mathbf{P})_{i, j} \sim \mathcal{B}(\mu=0, p=1 / 2)
$$

where $\mathcal{B}(\mu, p)$ denotes the Bernoulli distribution with mean $\mu$ and probability $p$. The resulting \pm 1 patterns are well suited to the ON/OFF states of the DMD.

Solution to $P_{2}$ : Under certain conditions such that $I \geq$ $O(K \log (P / K))$, the image can be exactly or closely recovered with a high probability [30] in the transform domain solving the following $\ell_{1}$-minimization problem:

$$
\mathbf{s}^{*}=\arg \min \|\mathbf{s}\|_{1} \text { such that } \Delta t \mathbf{P} \mathbf{\Lambda} \mathbf{s}=\mathbf{m} .
$$

This is a convex optimization problem that can be solved efficiently by different algorithms [55], [56]. The image is finally recovered in the original (image) domain according to (3), i.e.,

$$
\mathbf{f}^{*}=\Lambda \mathbf{s}^{*}
$$

Note that a popular alternative to (5) and (6) is to recover f directly in the image domain considering Total Variation minimization [57].

$$
\mathbf{f}^{*}=\arg \min \|\mathbf{f}\|_{\mathrm{TV}} \text { such that } \Delta t \mathbf{P f}=\mathbf{m} .
$$

\section{PRoposed ADAPTIVE BASIS SCAN BY WAVELET PREDICTION}

\section{A. Adaptive imaging}

The method we propose falls into the category of adaptive approaches. In such an iterative scheme, some of the patterns sent to the DMD are determined during the acquisition with a prediction step, as illustrated in Fig. 2. The acquisition starts with a predetermined set of patterns. The resulting measurements are exploited to predict a new set of patterns. When a given criterion is reached, the restoration of the image is performed.

In an adaptive approach, the image is acquired in a chosen basis. For instance, one can acquire an image with Fourier patterns, DCT patterns, wavelet patterns, etc. The adaptivity regards the elements of the chosen basis that are to be acquired based on the prediction method. The basis elements that are not acquired are simply discarded and set to 0 . The main advantage is that the image restoration is straightforward using the inverse transform of the chosen basis. This enables one to avoid the computational cost of $\ell_{1}$-minimization. The computational cost is shifted from the recovery to the prediction.

In this paper, we propose to obtain the measurements $\mathbf{m}$ of (1) from wavelet patterns $\mathbf{P}$ using a non-linear acquisition strategy and interpolation techniques. The wavelet transform has been chosen since it gives sparse signals thus allowing one to only acquire a small number of measurements $I<\angle P=N^{2}$.

\section{B. Wavelet decomposition}

The wavelet transform is a very powerful and popular tool [38], [39]. The discrete wavelet decomposition of an image $\mathbf{f} \in \mathbb{R}^{P \times 1}$ with the standard dyadic wavelets separates the signal into approximation and detail coefficients (horizontal, vertical or diagonal). The approximation coefficients result from a low-pass filtering, detail coefficients from a high-pass filtering [39].

Let $j=1 \ldots J$ be the scale [58] at which the image $\mathbf{f}$ is observed, $J$ being the (coarsest) decomposition level of the wavelet transform, with $1 \leq J \leq \log _{2}(N)=R$. A location is specified by the vector $\mathbf{k}$ so that

$$
\mathbf{k}=\left(k_{1}, k_{2}\right) \in\left\{1, \ldots, 2^{\ell}\right\}^{2} \quad \text { with } \quad \ell=R-j
$$

We note $\widetilde{\mathbf{f}}$ the wavelet transform of $\mathbf{f}$ :

$$
\tilde{\mathbf{f}}=\mathbf{W f}
$$

with $\mathbf{W} \in \mathbb{R}^{P \times P}$ an orthonormal operator [59]. $\tilde{\mathbf{f}} \in \mathbb{R}^{P \times 1}$ represents the image $\mathbf{f}$ in the wavelet domain and each of its element represents a wavelet coefficient. Each element may be fully identified and located by its unique triplet $i$ such that

$$
i=\{o, j, \mathbf{k}\}
$$

where $o=0,1,2$ or 3 represents the approximation, vertical, horizontal and diagonal coefficients, respectively. Each row of $\mathbf{W}$ corresponds to a unique triplet $i$. The image $\mathbf{f}$ can be perfectly recovered using the inverse wavelet transform:

$$
\mathbf{f}=\mathbf{W}^{-1} \widetilde{\mathbf{f}}
$$

The forward or inverse wavelet transform are widely used with fast algorithms implemented as filter banks [39].

This kind of decomposition was shown to give sparse signals, allowing one to discard many coefficients at the recovery step. An efficient approximation of the wavelet transform is the one where a number $I<<P$ of the largest coefficients are retained among all scales. The other coefficients are thresholded to 0 and the image restoration using (11) shows excellent image quality [39]. We will refer to this technique as the non-linear approximation. 


\section{Prediction strategy}

Our method ABS-WP is based on the non-linear approximation of the wavelet transform. Our goal is to acquire the significant wavelet coefficients and we therefore want to predict the triplets $i$ for each of these elements. The endgame is to fill the matrix $\mathbf{P}$ in (2) with the rows of $\mathbf{W}$ corresponding to the predicted triplet $i$ that we will note $\bar{i}$. In the case of the SPC, the whole wavelet transform of the object to be imaged is unknown. Therefore, we perform several non-linear approximations throughout the different scales of the wavelet decomposition. More precisely, our strategy decomposes into five steps. Step 1 works as an initialization whereas steps 2 to 4 are prediction steps and step 5 consists of the acquisition of the predicted significant wavelet coefficients:

1) The approximation image $\mathbf{A}_{j}$ at the lowest scale $j=J$ is fully acquired. This is a $2^{\ell} \times 2^{\ell}$ image with $\ell=R-j$. To be precise, this coarse image is acquired with the corresponding set of patterns at the approximation wavelet scale. This can therefore be seen as a basis scan acquiring the $2^{2 \ell}$ elements.

2) $\mathbf{A}_{j} \in \mathbb{R}^{2^{\ell} \times 2^{\ell}}$ is oversampled by a factor of two via an interpolation operator $\mathcal{S}$ to give $\mathbf{H}_{j}=\mathcal{S}\left(\mathbf{A}_{j}\right) \in \mathbb{R}^{2^{\ell+1} \times 2^{\ell+1}}$. Among many existing interpolation techniques, we used the bicubic interpolation [60] for its easy implementation and fast computation time.

3) The high resolution image $\mathbf{H}_{j}$ is one-level wavelet transformed to give $\widetilde{\mathbf{H}}_{j} \in \mathbb{R}^{2^{\ell+1} \times 2^{\ell+1}}$. This gives the predicted wavelet detail coefficients at scale $j$.

4) To predict the triplets $\bar{i}$ of the largest elements, we perform a non-linear approximation by retaining a percentage $p_{j}$ of the largest detail coefficients. This gives the predicted significant coefficients and their corresponding triplets $\bar{i}$.

5) The coefficients are then experimentally acquired sending the rows of $\mathbf{W}$ corresponding to $\bar{i}$ to the DMD.

For the other scales of the wavelet transform, steps 2 to 5 are unchanged. For the step 1 however, instead of the full acquisition of the approximation image at scale $j=J$, the approximation image $\mathbf{A}_{j}$ is obtained by the inverse wavelet transform of the coefficients acquired so far. For each level, a different value of $p_{j}$ is used giving the set of percentages

$$
\mathcal{P}=\left\{p_{J}, p_{J-1}, \ldots, p_{1}\right\} .
$$

Our strategy thus alternates between acquisition of the wavelet coefficients on the real image and prediction using an interpolation technique. Figure 3 presents a sketch of the algorithm of ABS-WP, the number for each step corresponds to the above steps.

\section{Compression rate}

The full acquisition of the approximation image $\mathbf{A}_{j}$ at scale $j=J$ leads to the acquisition of $n_{0}=2^{2 L}=4^{L}$ wavelet coefficients with $L=R-J$. Then, we acquire $p_{j}$ percent of the strongest predicted detail coefficients. Therefore the number of measurement at each scale $j$ is given by

$$
n_{j}=3 \times 2^{2^{l}} \times p_{j}=3 \times 4^{l} \times p_{j}
$$

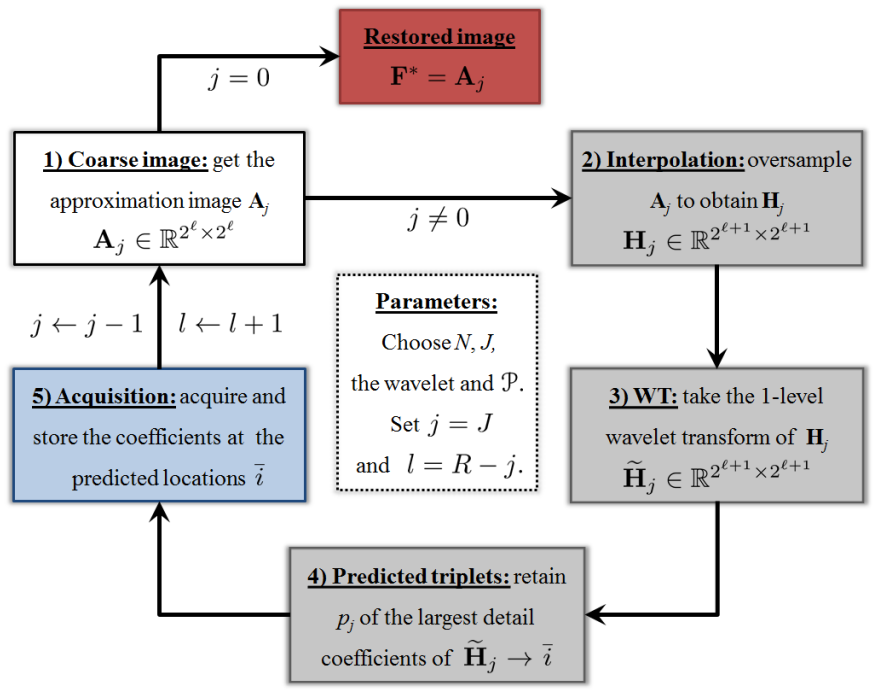

Fig. 3. Summary of the acquisition and prediction strategies of ABS-WP. White boxes corresponds to initialization or general processes, gray boxes to the prediction, the blue box is the acquisition step and the red one is the image restoration.

coefficients with $\ell=R-j$. We thus can control the total number of coefficients $n$ acquired for each decomposition level by modulating the set of percentages $\mathcal{P}$ in (12). Using (13), it can be shown that

$$
n=4^{L}\left[1+3 \sum_{j=1}^{J} 4^{J-j} p_{j}\right]
$$

We define the compression rate $(\mathrm{CR})$ as

$$
\mathrm{CR}=1-\frac{n}{P}
$$

which is a normalized quantity ranging from 0 to 1 . One can finally recover an image from the $n$ samples using the inverse wavelet transform.

\section{EXPERIMENTS OVERVIEW}

\section{A. Pattern generation}

To perform the acquisition, the patterns $\left\{\mathbf{p}_{i}\right\}$ that will be sent to the DMD have to be generated. One pattern can be obtained as:

$$
\mathbf{p}_{i}=\mathbf{W}^{-1} \mathbf{v}_{i}
$$

where $\mathbf{v}_{i}$ is a unit vector chosen from the natural basis $\left\{\mathbf{e}_{i}\right\}$. In practice, one can create a null image with only the pixel located at $i$ set to 1 , by taking the inverse wavelet transform of this image, one obtains the corresponding pattern for the triplet $i$.

Two practical problems now arise to send such images to the DMD: the obtained patterns have floating values and both negative and positive elements that cannot be physically implemented together on a DMD. To tackle the positivity problem, we divide $\mathbf{p}_{i}$ in its positive and absolute negative parts so that $\mathbf{p}_{i}=\mathbf{p}_{i}^{+}-\mathbf{p}_{i}^{-}$. Given the linearity of (1), the final measurement $m_{i}$ is obtained numerically as

$$
m_{i}=m_{i}^{+}-m_{i}^{-}
$$



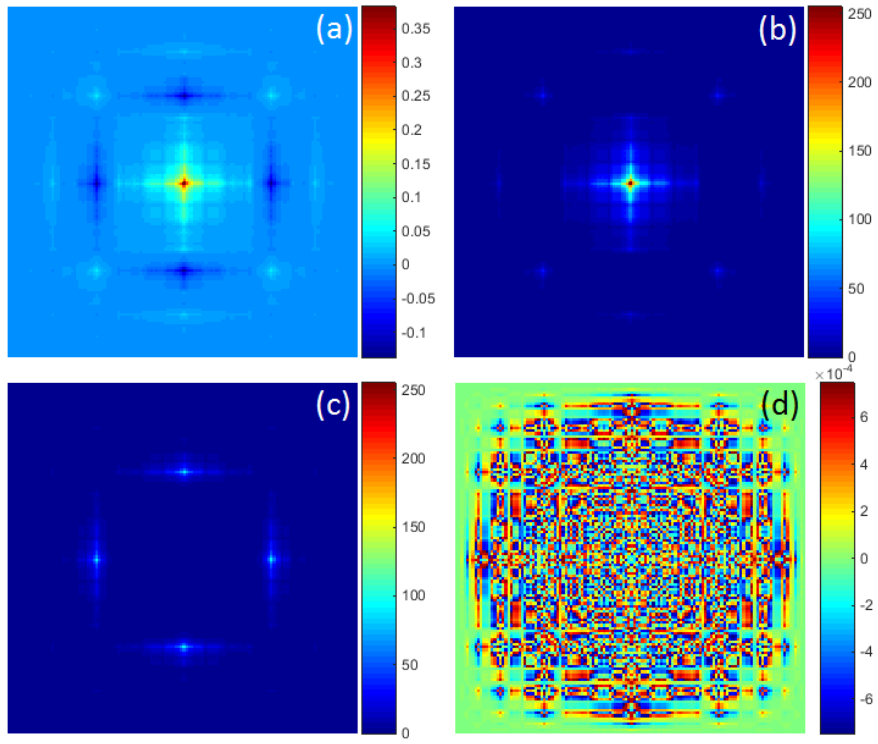

Fig. 4. Example of a wavelet pattern before and after quantization for $b=8$ bits using Le Gall wavelet. (a) Real wavelet pattern $\mathbf{p}_{i}$, (b) positive quantized part $\widehat{\mathbf{p}}_{i}^{+}$, (c) negative quantized part $\widehat{\mathbf{p}}_{i}^{-}$and (d) quantification error pattern $\widehat{\mathbf{e}}_{i}$.

where

$$
m_{i}^{+}=\Delta t \mathbf{f}^{\top} \mathbf{p}_{i}^{+} \quad \text { and } \quad m_{i}^{-}=\Delta t \mathbf{f}^{\top} \mathbf{p}_{i}^{-}
$$

are measured experimentally by the SPC.

Regarding the floating values of the patterns and to use any kind of wavelet, we perform uniform quantization to convert the patterns to $b$-bits patterns, $2^{b}$ being the maximum available dynamic range of the DMD. To realize this quantization, we apply the following transform to each pattern:

$$
\widehat{\mathbf{p}}_{i}=\left\lfloor\frac{1}{q_{i}} \mathbf{p}_{i}\right\rfloor \quad q_{i}=\frac{\max \left(\left|\mathbf{p}_{i}\right|\right)}{2^{b}-1}
$$

where $\lfloor$.$\rfloor denotes a rounding operation. Since the patterns$ depend on the triplet $i$ of (10), the quantization factor $q_{i}$ is also a function of the same triplet. We give an example of pattern in Fig. 4 using Le Gall (CDF 5/3) biorthogonal wavelet [61], [62]. The quantization leads to an irreversible loss of information depicted by the quantization error pattern $\widehat{\mathbf{e}}_{i}=q_{i} \widehat{\mathbf{p}}_{i}-\mathbf{p}_{i}$ as can be seen by Fig. 4-(d).

Assuming the quantization error can be neglected, the measurement $m_{i}$ in (17) can be obtained by

$$
m_{i}=q_{i}\left(\hat{m}_{i}^{+}-\hat{m}_{i}^{-}\right)
$$

where

$$
\hat{m}_{i}^{+}=\Delta t \mathbf{f}^{\top} \widehat{\mathbf{p}}_{i}^{+} \quad \text { and } \quad \hat{m}_{i}^{-}=\Delta t \mathbf{f}^{\top} \widehat{\mathbf{p}}_{i}^{-}
$$

are two measurements acquired by the SPC. Note that the value of $q_{i} \Delta t=\Delta t_{i}$ can directly be employed as an integration time to obtain correctly-scaled measurements:

$$
m_{i}^{+}=q_{i} \Delta t \mathbf{f}^{\top} \widehat{\mathbf{p}}_{i}^{+}=\Delta t_{i} \mathbf{f}^{\top} \widehat{\mathbf{p}}_{i}^{+} \quad \text { and } \quad m_{i}^{-}=\Delta t_{i} \mathbf{f}^{\top} \widehat{\mathbf{p}}_{i}^{-}
$$

are directly acquired by the SPC and the final measurement is numerically computed as in (17).

\section{B. Numerical experiments}

Different images have been used to perform several simulations. The well known image of Lena and the peppers image have been employed since they are commonly used in image processing. An optical microscopy image of vertebral bone tissue of a fetus shown in Fig. 5 serves as an indicator for textured images. Finally, fluorescence imaging being a target application, we consider the bioluminescence image of a mouse [63] shown in Fig. 6 superimposed to its ambient light image.

We compare our ABS-WP method to compressive imaging (CI) presented in Section II which is the reference nonadaptive approach. For CI simulations, instead of the $\ell_{1}$-minimization in (5), we directly reconstructed the image $\mathbf{f}$ from the measurements $\mathbf{m}$ using Total Variation (TV) minimization via TVAL3 [57] as done in [1], [2]. This is close to performing $\ell_{1}$-minimization in the wavelet domain [54] and it allows for much faster image restoration. Anisotropic TV with positivity was employed as it gave the best results in most cases.

We also compare our results to the adaptive method proposed by Dai [41]. In this adaptive method derived from Haar's wavelet, a threshold has to be chosen to decide the relevant coefficients to sample. For each image and compression rate, the threshold was tackled experimentally to obtain the best possible PSNR for the restored image.

In noisy simulations, the measurements are corrupted by Poisson noise. To do that, the noise was applied on the two measurements of (22) i.e. this equation becomes

$$
m_{i}^{+}=\mathscr{P}\left(q_{i} \Delta t \mathbf{f}^{\top} \widehat{\mathbf{p}}_{i}^{+}\right) \quad \text { and } \quad m_{i}^{-}=\mathscr{P}\left(q_{i} \Delta t \mathbf{f}^{\top} \widehat{\mathbf{p}}_{i}^{-}\right)
$$

where $\mathscr{P}$ is the Poisson distribution. Changing $\Delta t$ in (23) allows one, for a given image ff, to simulate several levels of noise by changing the number of average photons $N_{0}(\mathrm{ph})$ emitted by the object during $\Delta t$. The Poisson noise has indeed a greater effect when the values of $N_{0}$ are small.

\section{Experimental acquisitions}

To demonstrate the ability of our technique to work for real acquisitions, we performed several experiments. The experimental setup was composed of a supercontinuum pulsed laser source (SC-450, Fianium) followed by an IF filter with center wavelength at $650 \mathrm{~nm}$ for uniform illumination of the object. A $1024 \times 768$ DMD (DLP7000 - V7001, Vialux) was exploited to spatially modulate the image with a possibility of loading $b=8$-bits patterns by adjusting the duty cycle for each bit plane. The light reflected from the DMD is focused by means of a lens on a single pixel photomultiplier detector (HPM-10050, Becker \& Hickl GmbH). A time-correlated single photon counting (TCSPC) board (SPC-130, Becker \& Hickl GmbH) is also coupled to the photomultiplier. As a result, only timeresolved measurements are considered in this paper since we aim for applications such as fluorescence lifetime imaging in the future.

As an object, we chose two Jaszczak phantoms commonly used in CT. These different targets were printed on white paper and the obtained diameter was $22 \mathrm{~mm}$. The experimental CCD image of these targets can be seen in Fig. 7-(a) and Fig. 8-(a). 


\begin{tabular}{c|c|c|c|c|c|c}
\hline \hline \multirow{2}{*}{ Image } & \multicolumn{7}{|c}{ PSNR (dB) } \\
\cline { 2 - 7 } & $b=4$ & $b=6$ & $b=8$ & $b=10$ & $b=12$ & $b \rightarrow \infty$ \\
\hline $\begin{array}{c}\text { Bones } \\
(256 \times 256)\end{array}$ & 24.73 & 29.98 & 30.87 & 31.18 & 31.18 & 31.18 \\
\hline $\begin{array}{c}\text { Mouse } \\
(128 \times 128)\end{array}$ & 33.20 & 43.80 & 47.82 & 49.18 & 49.23 & 49.23 \\
\hline \hline
\end{tabular}

TABLE I

QUANTIZATION EFFECT IN OUR ABS-WP METHOD FOR LE GALL'S WAVELET FOR DIFFERENT NUMBER OF BITS $b$ FOR A CR OF $80 \%$. THE LAST COLUMN IS EQUIVALENT TO SIMULATE THE STRATEGY WITHOUT QUANTIZATION.

For each case, $128 \times 128$ pixels patterns were employed thus giving $128 \times 128$ pixels restored SPC images. The patterns were resized as $640 \times 640$ pixels patterns to use most of the DMD's height. This resizing operation was performed using a box-shaped kernel which means that no other pixel values other than those in the patterns were added. In other words, an area of $5 \times 5(640 / 128=5)$ DMD mirrors was used to represent one pixel of the $128 \times 128$ pattern.

\section{Integration time}

Concerning the integration time $\Delta t$ at the detector, it is, in theory, only limited by the DMD's frequency. As pointed before, a TCSPC is also employed in our setup. The use of the DMD at its highest speed in this case is limited by the statistics constraints of photon counting of the TCSPC. This requires integration time of a few hundred milliseconds to work properly while having a correct SNR in standard illumination conditions. In this particular case, the integration time $\Delta t$ at the single-pixel detector is always greater that what can be obtained without the TCSPC. As a result, in the following simulations and experimental results, $\Delta t$ was kept constant for each case so that the different techniques (CI, Dai's method or ABS-WP) are fairly comparable for a given number of measurements. Once performed, the measurements $\left\{\hat{m}_{i}\right\}$ of (21) were post-processed with their different quantization factor $\left\{q_{i}\right\}$ according to (20) to obtain correctly-scaled measurements.

\section{RESULts}

\section{A. Numerical experiments}

1) Influence of the quantization: Table I presents simulation results showing the quantization effect on two images with our method when using Le Gall's wavelet. An example of pattern using this wavelet can be seen in Fig. 4. The proposed strategy was simulated exactly as it would be computed by the SPC: the wavelet coefficients were obtained with the dot product between the corresponding quantized patterns and the image.

2) Influence of the prediction strategy: Table II presents the accuracy of the prediction strategy at identifying the significant wavelet coefficients for Dai's technique and our ABS-WP method. For each case presented, the Haar wavelet was employed since Dai's technique is derived from this wavelet. In order to only compare fairly the influence of the prediction, the set of percentages for each image for ABSWP was obtained from Dai's number of sampled wavelet

\begin{tabular}{c|c|c|c}
\hline \hline \multirow{2}{*}{ Image } & \multirow{2}{*}{$\mathbf{C R}$} & \multicolumn{2}{|c}{ Correctly matched wavelet coefficients (\%) } \\
\cline { 3 - 4 } & & Dai & $A B S-W P$ \\
\hline Bones & 80 & 62 & $\mathbf{6 9}$ \\
\cline { 2 - 4 }$(256 \times 256)$ & 85 & 59 & $\mathbf{6 5}$ \\
\hline Mouse & 80 & 82 & $\mathbf{8 5}$ \\
\cline { 2 - 4 }$(128 \times 128)$ & 85 & 79 & $\mathbf{8 4}$ \\
\hline \hline
\end{tabular}

TABLE II

ACCURACY OF THE PREDICTION STRATEGY FOR DAI'S TECHNIQUE AND OUR ABS-WP FRAMEWORK. THE TABLE DISPLAYS THE PERCENTAGES OF THE WAVELET COEFFICIENTS THAT WERE CORRECTLY PREDICTED AS SIGNIFICANT COMPARED TO THE TRUE SIGNIFICANT WAVELET COEFFICIENTS.

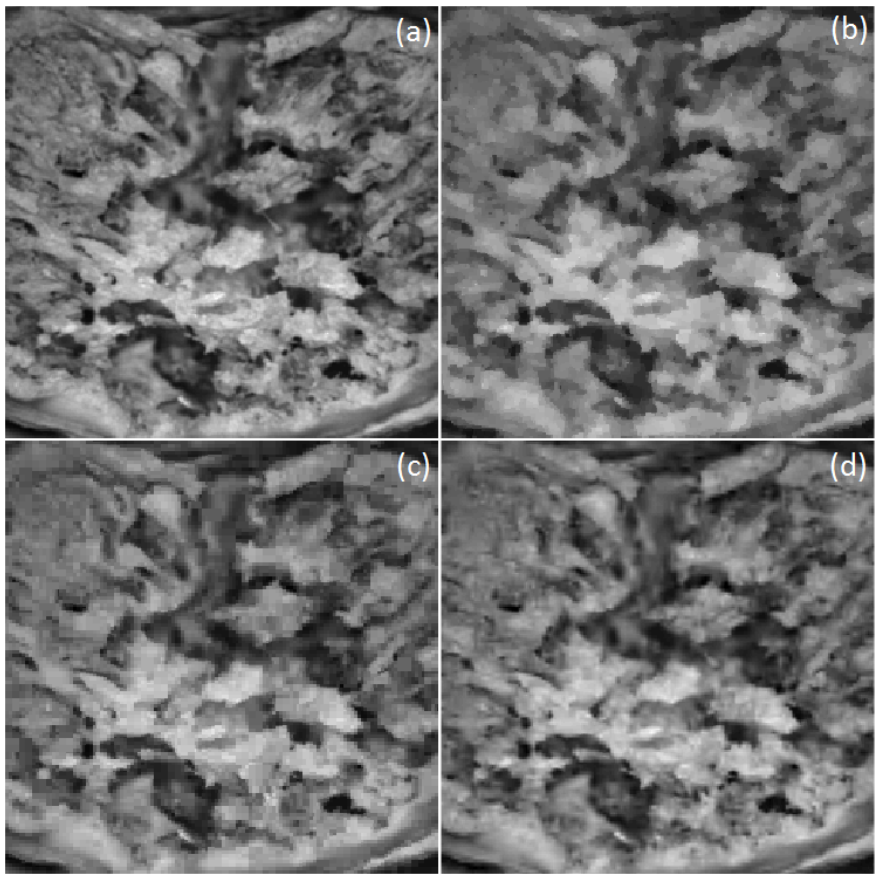

Fig. 5. Noise-free simulation of different SPC acquisition techniques on a $256 \times 256$ image of bones with a CR of $80 \%$. (a) Ground truth image, images restored with (b) CI, (c) Dai's method and (d) our ABS-WP technique. The PSNRs and parameters associated with these results are given in table III.

coefficients at each scale. Then, the locations of the found coefficients for both prediction strategies were compared to the true significant wavelet coefficients obtained from the ground truth images giving the percentages of accuracy in table II.

3) Influence of the acquisition strategy: Figure 5 gives simulated visual results of our method compared to CI and Dai's method for one test image. In the case of our method, Le Gall's wavelet was used since it proved to be the most efficient wavelet in several cases.

In table III, we present the obtained PSNRs for the different SPC acquisition techniques at two compression rates and table IV gives the associated average computation times.

4) Influence of the image: Four different images were considered: the well-known Lena and peppers images as well as the bone image depicted in Fig. 5 and the mouse fluorescence image depicted in Fig. 6. Note that the bone image is highfrequency while the mouse fluorescence is pretty smooth.

5) Influence of noise: Table V compares the performance of the different acquisitions strategies considering the noisy measurements as given by (23). Our ABS-WP method with 


\begin{tabular}{c|c|c|c|c||c}
\hline \hline \multirow{2}{*}{ Image } & \multirow{2}{*}{ CR } & \multicolumn{3}{|c||}{ PSNR $(\mathrm{dB})$} & $\begin{array}{c}\text { Dai's } \\
\text { thresholds }\end{array}$ \\
\cline { 3 - 6 } & & $C I$ & Dai & $A B S-W P$ & 11.52 \\
\hline \multirow{2}{*}{ Lena $(256 \times 256)$} & 80 & 29.55 & 29.90 & $\mathbf{3 0 . 3 3}$ & 16.63 \\
\cline { 2 - 6 } & 85 & 27.89 & 28.49 & $\mathbf{2 9 . 5 9}$ & 7.71 \\
\hline \multirow{2}{*}{ Peppers $(256 \times 256)$} & 80 & 34.70 & 35.06 & $\mathbf{3 5 . 3 5}$ & 11.77 \\
\cline { 2 - 6 } & 85 & 32.96 & 33.42 & $\mathbf{3 4 . 8 3}$ & 12.89 \\
\hline \multirow{2}{*}{ Bones $(256 \times 256)$} & 80 & 29.38 & 30.24 & $\mathbf{3 1 . 1 8}$ & 17.61 \\
\cline { 2 - 6 } & 85 & 28.14 & 28.59 & $\mathbf{3 0 . 2 9}$ & 385.65 \\
\hline \multirow{2}{*}{ Mouse $(128 \times 128)$} & 80 & 45.36 & 47.65 & $\mathbf{4 9 . 2 3}$ & 851.10 \\
\cline { 2 - 6 } & 85 & 42.18 & 45.83 & $\mathbf{4 9 . 1 3}$ & \\
\hline \hline
\end{tabular}

TABLE III

OBTAINED PSNRS FOR DIFFERENT SPC ACQUISITION TECHNIQUES AT TWO COMPRESSION RATES ON SEVERAL TEST IMAGES IN A NOISE-FREE SETTING. THE THRESHOLDS USED FOR DAI'S METHOD ARE GIVEN IN THE LAST COLUMN. FOR ABS-WP, LE GALL PATTERNS WERE EMPLOYED WITH $\mathcal{P}=\{0.90,0.80,0.71,0.02\}$ AND $\mathcal{P}=\{0.90,0.80,0.45,0.019\}$ TO GIVE CRS OF $80 \%$ AND $85 \%$.

\begin{tabular}{c|c|c|c|c}
\hline \hline \multirow{2}{*}{ Image size } & \multirow{2}{*}{ CR } & \multicolumn{3}{|c}{ Time (s) } \\
\cline { 3 - 5 } & & $C I$ & Dai & $A B S-W P$ \\
\hline \multirow{2}{*}{$256 \times 256$} & 80 & 267.37 & $\mathbf{0 . 1 2}$ & 0.43 \\
\cline { 2 - 5 } & 85 & 213.62 & $\mathbf{0 . 0 9}$ & 0.42 \\
\hline \multirow{2}{*}{$128 \times 128$} & 80 & 15.50 & $\mathbf{0 . 0 2}$ & 0.19 \\
\cline { 2 - 5 } & 85 & 13.18 & $\mathbf{0 . 0 2}$ & 0.18 \\
\hline \hline
\end{tabular}

TABLE IV

AVERAGE COMPUTATION TIME FOR THE DIFFERENT SPC ACQUISITION TECHNIQUES FOR THE RESULTS OF TABLE III. THE TIME INCLUDES THE IMAGE RESTORATION FOR CI AND PREDICTION + RESTORATION FOR DAI'S METHOD AND OUR TECHNIQUE.

two different wavelets is compared to Dai's technique and compressive imaging on the Fig. 6-(a) for different values of $\Delta t$ i.e. different levels of noise. A low $\Delta t$ leads to a low average of photons $N_{0}$ emitted by the object and thus the noise effect is greater.

\section{B. Experimental acquisitions}

Figure 7 presents real SPC acquisitions of a target with our acquisition strategy (ABS-WP) and compressive imaging as a comparison. In the case of ABS-WP, we used both Haar and Le Gall wavelets to show the ability of the DMD to use 8-bits patterns.

Figure 8 allows to judge the ability of our optical setup to discern small dots at different compression rates. The printed dots diameters are about $1 \mathrm{~mm}$ for the smallest dots and about $3 \mathrm{~mm}$ for the biggest ones. A pixel size of $210 \mu \mathrm{m}$ was measured in our setup that can be improved by changing optics and/or change the patterns' size.

\begin{tabular}{c|c|c|c|c|c}
\hline \hline \multirow{2}{*}{$\Delta t(\mathrm{~s})$} & \multirow{2}{*}{$N_{0}(p h)$} & \multicolumn{4}{|c}{ PSNR (dB) } \\
\cline { 3 - 6 } & & $C I$ & $D a i$ & $A B S-W P($ Le G. $)$ & $A B S-W P($ Haar $)$ \\
\hline 1 & 5245 & 38.99 & 45.72 & $\mathbf{4 7 . 2 0}$ & 46.05 \\
\hline 0.75 & 3934 & 39.29 & 45.61 & $\mathbf{4 6 . 8 8}$ & 45.98 \\
\hline 0.5 & 2623 & 39.04 & 45.48 & $\mathbf{4 6 . 5 4}$ & 45.91 \\
\hline 0.25 & 1312 & 38.47 & 45.31 & $\mathbf{4 5 . 9 0}$ & 45.71 \\
\hline 0.1 & 525 & 37.06 & 44.72 & 43.99 & $\mathbf{4 5 . 0 1}$ \\
\hline \hline
\end{tabular}

TABLE V

NOISY SIMULATIONS FOR DIFFERENT ACQUISITION STRATEGIES AT A CR OF $85 \%$ FOR DIFFERENT VALUES OF $\Delta t$ ON THE MOUSE IMAGE OF

Fig. 6-(A). FOR ABS-WP, THE SET OF PERCENTAGES USED FOR LE GALL'S WAVELET WAS $\mathcal{P}=\{0.90,0.80,0.45,0.019\}$ AND $\mathcal{P}=\{0.77,0.42,0.24,0.1\}$ FOR HAAR

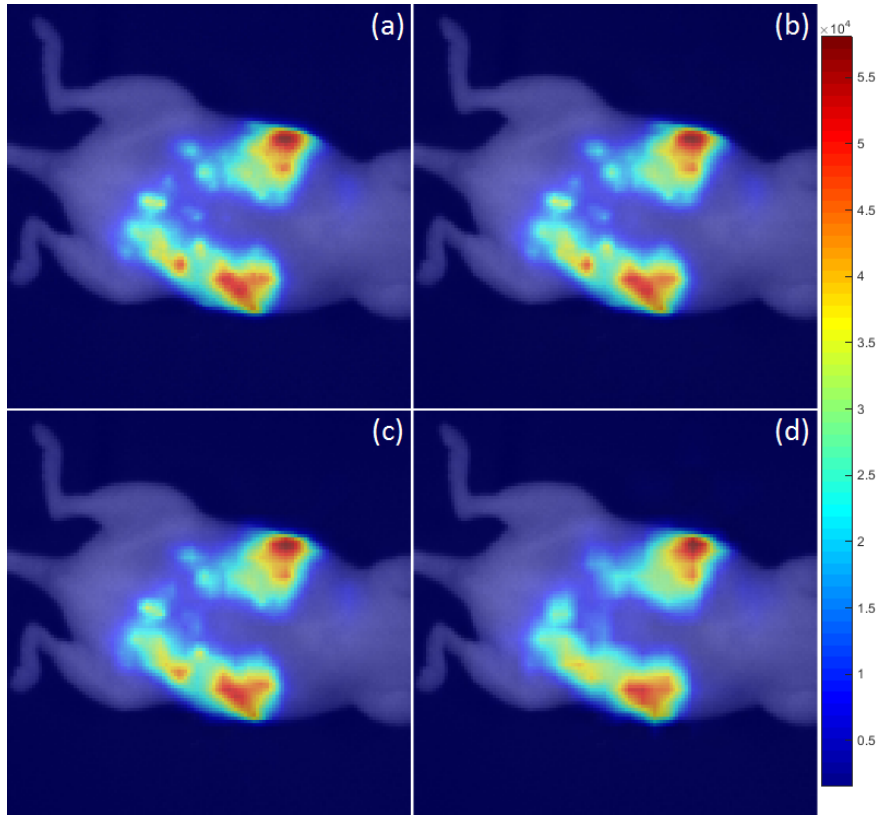

Fig. 6. Noise-free simulation of our acquisition strategy on a $128 \times 128$ bioluminescence image of a mouse. The bioluminescence images have been overlaid on the ambient light image of the mouse. (a) Ground truth image, images restored using Le Gall's wavelet for a CR of (b) $90 \%$, (c) $95 \%$ and (d) $98 \%$. Respectively, PSNRs compared to the ground truth image are 48.25 $\mathrm{dB}, 41.48 \mathrm{~dB}$ and $35.37 \mathrm{~dB}$

\section{DISCUSSION}

Our ABS-WP strategy presented in Section III was designed to overcome the $\ell_{1}$-minimization of CS by acquiring an image in a wavelet basis. In addition, non-linear approximations are employed to avoid image-dependent thresholds. In order to use any possible wavelet, uniform quantization of the patterns is performed.

Haar wavelet is often considered [40]-[43] since, up to a scale factor, the patterns have only 0 or 1 values and the quantization therefore does not impact the image quality. However in the case of our ABS-WP technique, as can be seen in table I, this quantization impacts the quality of the restored image when another wavelet is employed. With a CR of $80 \%$ and for values of $b \leq 10$ the restored images have a smaller PSNR than the one recovered with real patterns (last column, $b \rightarrow \infty$ ). This difference clearly comes from the rounding operation in (19) and is irreversible. When $b \geq 10$, this extends the grayscale and we can see that the quantization error can be considered negligible. As mentioned before, the quantization factor $q_{i}$ impacts the effective integration time $\Delta t_{i}=q_{i} \Delta t$ at the detector. For the proposed experiments in this paper, we always kept $\Delta t$ constant and post-processed the measurements by applying $q_{i}$ as depicted in (20) and (21). This is not the finest possible solution to obtain the best possible SNR. The optimum solution would be to increase or decrease the integration time according to the pattern i.e. $\Delta t_{i}=q_{i} \Delta t$ would act as the new integration time as in (22).

As mentioned previously, the bicubic interpolation was used for our acquisition strategy. This choice was based on several experiments with different existing interpolation and superresolution techniques [64]. This is surprising since the bicubic 


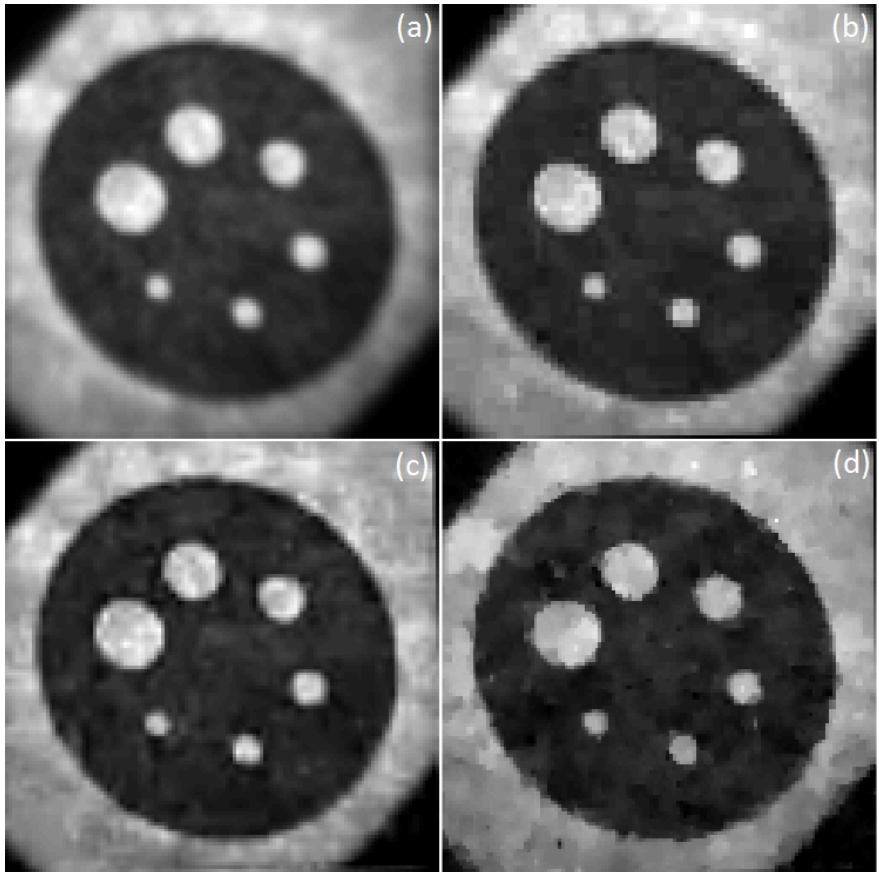

Fig. 7. Experimental acquisitions with the SPC on the Jaszczak target. (a) Experimental CCD image of the printed target on a paper, recovered $128 \times 128$ pixels images with a CR of $85 \%$ (b) for ABS-WP with Haar, (c) for ABS-WP with Le Gall and (d) using CI. Respectively, the obtained PSNRs compared to the CCD image after registration are $21.99 \mathrm{~dB}, 21.65 \mathrm{~dB}$ and $21.20 \mathrm{~dB}$. The dynamic of the SPC images has been rescaled to the dynamic range of the CCD image for visual comparison.

interpolation tends to smooth edges in general. We could assume that the location of the significant coefficients should be better predicted with more sophisticated techniques as in general, the highest wavelet coefficients are in the vicinity of the edges [39]. In spite of the smoothness of the bicubic interpolation, the technique gives very good results to predict the significant coefficient locations. This is confirmed by the results of table II where our technique is compared to Dai's. It can be seen that our prediction technique performs better at finding the true significant wavelet coefficients compared to Dai's thresholding technique. The results of table II where provided for Haar's wavelet since Dai's method is derived from it. However, in the case of ABS-WP, the possibility to use another wavelet can greater improve the image quality provided that the wavelet was correctly chosen.

Looking at the results of table III where we compare our method with CI or Dai's method it can be noted that we obtain numerically close or better results. As can be seen in Fig. 5, the TV-minimization leads to the creation of spot patterns when the image has lots of details and/or textures. Dai's method, because of the use of Haar's wavelet, shows pixelation that is not present in our technique with Le Gall's wavelet. The computation time in table IV also shows the improvement when an adaptive approach is considered. This improvement is again greater when bigger images are considered. Dai's method is extremely fast since the prediction is simply based on thresholding.

The thresholds for Dai's method presented in table III reveals that they are image-dependent and should be adjusted

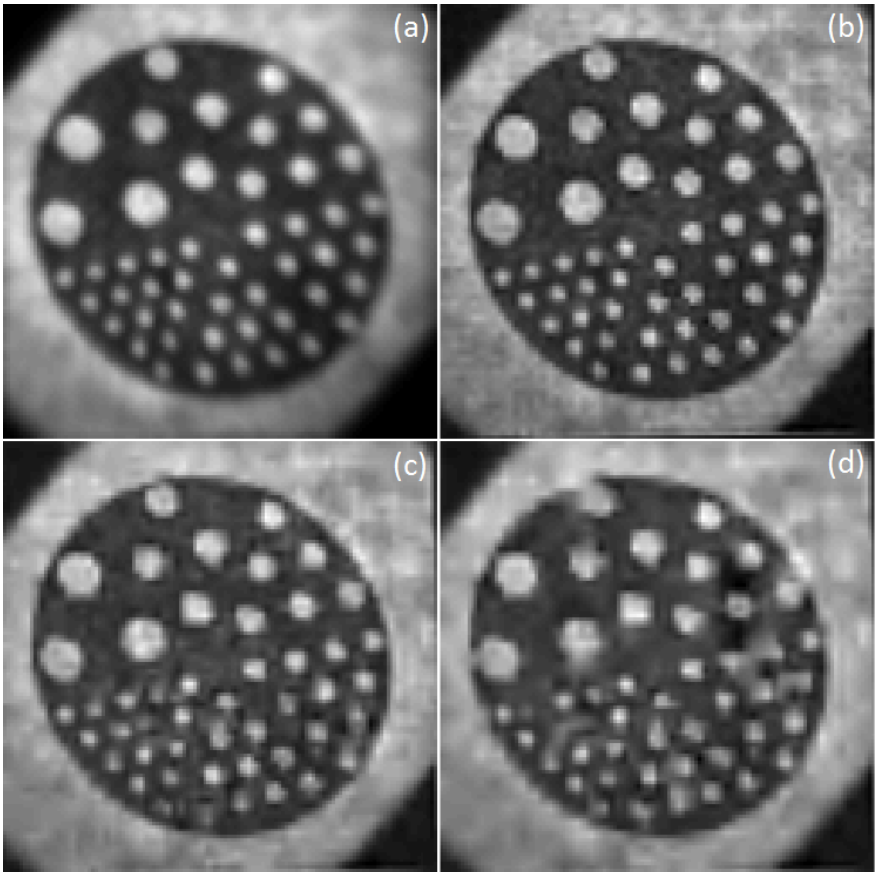

Fig. 8. Ability of the system to distinguish dots whose diameters range from $1 \mathrm{~mm}$ to $3 \mathrm{~mm}$. (a) Experimental CCD image of the printed target on a paper, recovered $128 \times 128$ pixels images with ABS-WP with Le Gall for a CR of (b) $75 \%$, (c) $85 \%$ and (d) $90 \%$. Respectively, the obtained PSNRs compared to the CCD image after registration are $22.35 \mathrm{~dB}, 21.51 \mathrm{~dB}$ and $20.85 \mathrm{~dB}$. The dynamic of the SPC images has been rescaled to the dynamic range of the CCD image for visual comparison. A pixel size of $210 \mu \mathrm{m}$ was measured.

for each image. In comparison, for our technique and a fixed $\mathrm{CR}$, the same set of percentages was used for each image. Despite the clear difference of the four involved images of table III, our strategy restores good quality images. This shows that ABS-WP adapts to the image. In our case, the different sets of percentages have been set once and for all after learning from several test images. In practice, one can use in simulation the non-linear approximation for a given CR on several images and find the number of retained coefficients in each level $j$. The average of the obtained values between the images gives a good candidate for the set of percentage $\mathcal{P}$. In the case of CI with TV-minimization, many parameters have to be tuned. The quality of the restored image dramatically depends on these choices. It was found that anisotropic TV with positivity constraint gave the best results.

Figure 6 demonstrates that even with a $\mathrm{CR}$ as high as 98\% one can recover an excellent image in the case of smooth images. For smooth images such as this one, only a few wavelet coefficients are needed to restore the principal features. The value of $J$ can thus be set closed to the limit $\log _{2}(N)$ and the percentages $p_{j}$ for small values of $j$ can be set to 0 . Such images indeed have very few details, only the coarser coefficients are sufficient enough to restore an image. On the contrary, for images with high frequency components, one should chose high values for the percentage $p_{1}$ to acquire the finest details. The choice of the set of percentages $\mathcal{P}$ and the decomposition level $J$ is therefore linked to the type of object to image and the aimed application.

The noisy simulations of table $\mathrm{V}$ show that our technique 
ABS-WP still perform close or better than compressive imaging or Dai's technique in a real-world setting. However, we note that the measurements are more rapidly corrupted by noise in the case of our method with Le Gall's patterns. This is because such patterns reflect less light than Haar patterns. The measurements of (23) are therefore smaller for Le Gall than Haar leading to a greater effect of the Poisson noise for the biorthogonal wavelet. In really low-light scenarios, one should in this case better use the simple Haar wavelet (last row of table V). In the future, we plan to propose a strategy to modify any wavelet patterns and reduce the effect of the noise in such cases.

If we move on to the experimental results, Figure 7 proves that, as Haar's wavelet, a more sophisticated wavelet such as Le Gall's can be used for acquisition. Visually, Le Gall gives a better result with a smoother image. The CI creates visible spots on the restored image. For Haar, the pixelation arises since $p_{1}$ was set to a very small value. The choice of the wavelet is also an important feature of our strategy. Depending on the object to image, some wavelets are better at sensing the scene than others i.e. they better capture the information in fewer coefficients, giving a sparser wavelet transform in one wavelet basis compared to another. For instance if a very smooth object is considered, a Battle wavelet would be much more appropriate than a Haar wavelet and fewer Battle coefficients would be needed compared to using Haar wavelet. With ABS-WP, one can choose any wavelet best adapted to the desired application and object to be acquired.

Finally, Figure 8 provides some insights about the system's possibilities. It can be seen that in our actual configuration, the measurements can discern objects of at least $1 \mathrm{~mm}$ provided that the compression rate is well chosen. For instance, for a CR of $80 \%$ or $85 \%$ one can discern the small dots. However, for $90 \%$, not enough elements have been sampled to restore the dots. In the case of our method, one can easily keep the acquisition going by lowering the compression rate after a first acquisition if the quality of the image is not judged sufficient. We can indeed keep on filling the wavelet transform of our image by adding new wavelet coefficients and quickly obtain a new restored image by inverse wavelet transform.

Some limitations of our ABS-WP framework deals with the employment of 8-bit (or more) patterns that reduces the maximum possible acquisition frame rate. For instance, the DMD in our setup can work at a maximum frequency of 22 $\mathrm{kHz}$ in 1-bit mode but only $290 \mathrm{~Hz}$ in 8-bit. This means that, by employing 8-bit wavelet patterns in our ABS-WP technique, one is not able to work at the highest frame rate. Depending on the application, this can be a problem. However, there are many different issues which practically limit the possibility to reach the DMD frame rate such as the weak SNR in low-light scenarios. This is particularly true in the field of biomedical optics where, e.g., fluorescence signal emitted by specific dyes are weak. Based on the results of Fig. 7 and table $\mathrm{V}$, in any case, if fast measurements are required, one can still use Haar's wavelet with our framework and obtain better results compared to CI or Dai's method thanks to a better prediction (table II).

Overall, ABS-WP needs few parameters making it a fast, easy to adjust and threshold-free adaptive acquisition technique. Unlike the CS approach, the perfect recovery of the signal is not guaranteed in theory unless each wavelet coefficient is acquired. It is however easy to refine the recovered image for ABS-WP by making a second pass of the algorithm. In the case of CS, doing such a process is time consuming because of the TV-minimization that would have to be started from scratch again. With ABS-WP, this simply allows one to complete the previously obtained wavelet transform of the image by sampling new coefficients.

\section{CONCLUSION}

We presented a new framework for single-pixel camera imaging. The philosophy of our approach is inspired by the non-linear approximation of the wavelet transform. It uses an interpolation technique to predict the significant wavelet coefficients that have to be experimentally acquired, while the other coefficients can be discarded. The main advantage of the proposed adaptive wavelet approach is to dispose of the computational overhead of $\ell_{1}$-minimization required by the compressed sensing theory. To our knowledge, this is the first time that a wavelet other than Haar's is used for experimental data in an adaptive strategy for SPC. Employing more sophisticated wavelets is made possible by uniform quantization of the wavelet patterns and allows one to choose the best suited wavelet for the desired application. Simulations and experimental acquisitions with the proposed methodology show both good visual and quantitative results and the method was proven to adapt to different kind of images.

The SPC opens many perspectives in the biomedical field. In future work, we plan to use this optical setup to perform time-resolved fluorescence imaging of biological structures.

\section{ACKNOWLEDGMENTS}

The authors thank Jean-Luc Coll and Veronique Josserand (Université Joseph Fourier, Institut Albert Bonniot INSERM U1209) for providing the ground truth image of Fig. 6 used to perform simulations.

\section{REFERENCES}

[1] D. Takhar, J. N. Laska, M. B. Wakin, M. F. Duarte, D. Baron, S. Sarvotham, K. F. Kelly, and R. G. Baraniuk, "A new compressive imaging camera architecture using optical-domain compression," in in Proc. of Computational Imaging IV at SPIE Electronic Imaging, 2006, pp. 43-52.

[2] M. Duarte, M. Davenport, D. Takhar, J. Laska, T. Sun, K. Kelly, and R. Baraniuk, "Single-pixel imaging via compressive sampling," Signal Processing Magazine, IEEE, vol. 25, no. 2, pp. 83-91, March 2008.

[3] R. H. Hadfield, "Single-photon detectors for optical quantum information applications," Nature photonics, vol. 3, no. 12, pp. 696-705, 2009.

[4] S. L. Jacques, "Optical properties of biological tissues: a review," Physics in Medicine and Biology, vol. 58, no. 11, p. R37, 2013.

[5] J. Ma, "Single-pixel remote sensing," IEEE Geoscience and Remote Sensing Letters, vol. 6, no. 2, pp. 199-203, April 2009.

[6] — "A single-pixel imaging system for remote sensing by two-step iterative curvelet thresholding," IEEE Geoscience and Remote Sensing Letters, vol. 6, no. 4, pp. 676-680, Oct 2009.

[7] J. Shin, B. T. Bosworth, and M. A. Foster, "Single-pixel imaging using compressed sensing and wavelength-dependent scattering," Opt. Lett., vol. 41, no. 5, pp. 886-889, Mar 2016.

[8] A. Rogalski, "History of infrared detectors," Opto-Electronics Review, vol. 20 , no. 3 , pp. 279-308, 2012. 
[9] S. S. Welsh, M. P. Edgar, R. Bowman, P. Jonathan, B. Sun, and M. J. Padgett, "Fast full-color computational imaging with single-pixel detectors," Opt. Express, vol. 21, no. 20, pp. 23 068-23 074, Oct 2013.

[10] B. Sun, M. P. Edgar, R. Bowman, L. E. Vittert, S. Welsh, A. Bowman, and M. J. Padgett, " $3 \mathrm{~d}$ computational imaging with single-pixel detectors," Science, vol. 340, no. 6134, pp. 844-847, 2013.

[11] J. H. Shapiro, "Computational ghost imaging," Phys. Rev. A, vol. 78, p. 061802, Dec 2008.

[12] V. Studer, J. Bobin, M. Chahid, H. Shams Mousavi, E. Candes, and M. Dahan, "Compressive fluorescence microscopy for biological and hyperspectral imaging," in Proceedings of the National Academy of Sciences of the USA, vol. 109, no. 26, 2012, pp. E1679-E1687.

[13] F. Magalhaes, M. Abolbashari, F. M. Araujo, M. V. Correia, and F. Farahi, "High-resolution hyperspectral single-pixel imaging system based on compressive sensing," Optical Engineering, vol. 51, no. 7, pp. 071 406-1-071 406-6, 2012.

[14] L. Bian, J. Suo, G. Situ, Z. Li, J. Fan, F. Chen, and Q. Dai, "Multispectral imaging using a single bucket detector," Scientific Reports, vol. 6, no. 24752, 2016.

[15] Q. Pian, R. Yao, N. Sinsuebphon, and X. Intes, "Hyperspectral compressive single-pixel imager for fluorescence lifetime sensing," in Biomedical Optics 2016. Optical Society of America, 2016, p. OTu2C.7.

[16] W. L. Chan, K. Charan, D. Takhar, K. F. Kelly, R. G. Baraniuk, and D. M. Mittleman, "A single-pixel terahertz imaging system based on compressed sensing," Applied Physics Letters, vol. 93, no. 12, 2008.

[17] C. M. Watts, D. Shrekenhamer, J. Montoya, G. Lipworth, J. Hunt, T. Sleasman, S. Krishna, D. R. Smith, and W. J. Padilla, "Terahertz compressive imaging with metamaterial spatial light modulators," Nature Photonics, vol. 8, no. 8, pp. 605-609, 2014.

[18] M. P. Edgar, G. M. Gibson, R. W. Bowman, B. Sun, N. Radwell, K. J. Mitchell, S. S. Welsh, and M. J. Padgett, "Simultaneous real-time visible and infrared video with single-pixel detectors," Scientific reports, vol. 5, 2015.

[19] Y. Zhang, M. P. Edgar, B. Sun, N. Radwell, G. M. Gibson, and M. J. Padgett, "3d single-pixel video," Journal of Optics, vol. 18, no. 3, p. 035203, 2016

[20] W. Becker, "Fluorescence lifetime imaging techniques and applications," Journal of Microscopy, vol. 247, no. 2, pp. 119-136, 2012.

[21] N. Radwell, K. J. Mitchell, G. M. Gibson, M. P. Edgar, R. Bowman, and M. J. Padgett, "Single-pixel infrared and visible microscope," Optica, vol. 1, no. 5, pp. 285-289, Nov 2014.

[22] A. Rodriguez, P. Clemente, E. Tajahuerce, and J. Lancis, "Dual-mode optical microscope based on single-pixel imaging," Optics and Lasers in Engineering, vol. 82, pp. 87 - 94, 2016.

[23] E. Tajahuerce, V. Durán, P. Clemente, E. Irles, F. Soldevila, P. Andrés, and J. Lancis, "Image transmission through dynamic scattering media by single-pixel photodetection," Opt. Express, vol. 22, no. 14, pp. 1694516955, Jul 2014.

[24] V. Duran, F. Soldevila, E. Irles, P. Clemente, E. Tajahuerce, P. Andrés, and J. Lancis, "Compressive imaging in scattering media," Opt. Express, vol. 23, no. 11, pp. 14424-14433, Jun 2015.

[25] A. Gibson and H. Dehghani, "Diffuse optical imaging," Philosophical Transactions of the Royal Society of London A: Mathematical, Physical and Engineering Sciences, vol. 367, no. 1900, pp. 3055-3072, 2009.

[26] C. D'Andrea, N. Ducros, A. Bassi, S. Arridge, and G. Valentini, "Fast 3d optical reconstruction in turbid media using spatially modulated light," Biomed. Opt. Express, vol. 1, no. 2, pp. 471-481, 2010.

[27] N. Ducros, A. Bassi, G. Valentini, G. Canti, S. Arridge, and C. D'Andrea, "Fluorescence molecular tomography of an animal model using structured light rotating view acquisition," Journal of Biomedical Optics, vol. 18, no. 2, pp. 020 503-020 503, 2013.

[28] Q. Pian, R. Yao, L. Zhao, and X. Intes, "Hyperspectral time-resolved wide-field fluorescence molecular tomography based on structured light and single-pixel detection," Optics Letters, vol. 40, pp. 431-434, 2015.

[29] N. Ducros, T. Correia, A. Bassi, G. Valentini, S. Arridge, and C. D'Andrea, "Reconstruction of an optical inhomogeneity map improves fluorescence diffuse optical tomography," Biomedical Physics \& Engineering Express, vol. 2, no. 5, p. 055020, 2016.

[30] D. L. Donoho, "Compressed sensing," IEEE Trans. Inform. Theory, vol. 52, pp. 1289-1306, 2006.

[31] R. M. Willett, R. F. Marcia, and J. M. Nichols, "Compressed sensing for practical optical imaging systems: a tutorial," Optical Engineering, vol. 50, no. 7, pp. 072 601-072601-13, 2011.

[32] R. F. Marcia, R. M. Willett, and Z. T. Harmany, Compressive Optical Imaging: Architectures and Algorithms. Wiley- $\mathrm{VCH}$ Verlag $\mathrm{GmbH} \&$ Co. KGaA, 2011, pp. 485-505.
[33] F. Magalhaes, F. M. Araujo, M. V. Correia, M. Abolbashari, and F. Farahi, "Active illumination single-pixel camera based on compressive sensing," Appl. Opt., vol. 50, no. 4, pp. 405-414, Feb 2011.

[34] R. A. DeVerse, R. R. Coifman, A. C. Coppi, W. G. Fateley, F. Geshwind, R. M. Hammaker, S. Valenti, F. J. Warner, and G. L. Davis, "Application of spatial light modulators for new modalities in spectrometry and imaging," in Spectral Imaging: Instrumentation, Applications, and Analysis II, vol. 4959, Jul. 2003, pp. 12-22.

[35] G. Davis, M. Maggioni, F. Warner, F. Geshwind, A. Coppi, R. DeVerse, and R. Coifman, "Hyperspectral analysis of normal and malignant microarray tissue sections using a novel micro-optoelectrical-mechanical system," Modern Pathology, vol. 17, no. Supplement 1, p. 358A, 2004.

[36] S. S. Welsh, M. P. Edgar, R. Bowman, B. Sun, and M. J. Padgett, "Near video-rate linear stokes imaging with single-pixel detectors," Journal of Optics, vol. 17, no. 2, p. 025705, 2015.

[37] Z. Zhang, X. Ma, and J. Zhong, "Single-pixel imaging by means of fourier spectrum acquisition," Nature communications, vol. 6, 2015.

[38] D. S. Taubman and M. W. Marcellin, JPEG 2000: Image Compression Fundamentals, Standards and Practice. Norwell, MA, USA: Kluwer Academic Publishers, 2001

[39] S. Mallat, A Wavelet Tour of Signal Processing, Third Edition: The Sparse Way, 3rd ed. Academic Press, Dec. 2008.

[40] S. Deutsch, A. Averbush, and S. Dekel, "Adaptive compressed image sensing based on wavelet modeling and direct sampling," in SAMPTA'09, Laurent Fesquet and Bruno Torrésani, Ed., Marseille, France, May 2009, p. General session.

[41] H. Dai, G. Gu, W. He, F. Liao, J. Zhuang, X. Liu, and Q. Chen, "Adaptive compressed sampling based on extended wavelet trees," Appl. Opt., vol. 53, no. 29, pp. 6619-6628, Oct 2014.

[42] A. Averbuch, S. Dekel, and S. Deutsch, "Adaptive compressed image sensing using dictionaries," SIAM Journal on Imaging Sciences, vol. 5 , no. 1, pp. 57-89, 2012.

[43] W.-K. Yu, M.-F. Li, X.-R. Yao, X.-F. Liu, L.-A. Wu, and G.-J. Zhai, "Adaptive compressive ghost imaging based on wavelet trees and sparse representation," Opt. Express, vol. 22, no. 6, pp. 7133-7144, Mar 2014.

[44] J. Hahn, C. Debes, M. Leigsnering, and A. M. Zoubir, "Compressive sensing and adaptive direct sampling in hyperspectral imaging," Digital Signal Processing, vol. 26, pp. 113 - 126, 2014

[45] F. Soldevila, E. Salvador-Balaguer, P. Clemente, E. Tajahuerce, and J. Lancis, "High-resolution adaptive imaging with a single photodiode," Scientific Reports, no. 5, 092015.

[46] W. Pratt, J. Kane, and H. C. Andrews, "Hadamard transform image coding," Proceedings of the IEEE, vol. 57, no. 1, pp. 58-68, Jan 1969.

[47] P. Indyk, E. Price, and D. P. Woodruff, "On the power of adaptivity in sparse recovery," in Foundations of Computer Science (FOCS), 2011 IEEE 52nd Annual Symposium on, Oct 2011, pp. 285-294.

[48] M. A. Davenport and E. Arias-Castro, "Compressive binary search," in Information Theory Proceedings (ISIT), 2012 IEEE International Symposium on, July 2012, pp. 1827-1831.

[49] M. L. Malloy and R. D. Nowak, "Near-optimal adaptive compressed sensing," IEEE Transactions on Information Theory, vol. 60, no. 7, pp. 4001-4012, July 2014.

[50] F. Rousset, N. Ducros, C. D'Andrea, and F. Peyrin, "Single pixel camera: An acquisition strategy based on the non-linear wavelet approximation," in 2015 37th Annual International Conference of the IEEE Engineering in Medicine and Biology Society (EMBC), Aug 2015, pp. 6240-6243.

[51] [Online]. Available: http://www.creatis.insa-lyon.fr/ ducros/single pixel_imaging

[52] F. Rousset, N. Ducros, A. Farina, G. Valentini, C. D'Andrea, and F. Peyrin, "Adaptive acquisitions in biomedical optical imaging based on single pixel camera: Comparison with compressive sensing," in 2016 IEEE 13th International Symposium on Biomedical Imaging (ISBI), April 2016, pp. 680-683.

[53] R. Baraniuk, M. Davenport, R. Devore, and M. Wakin, "A simple proof of the restricted isometry property for random matrices," Constr. Approx, vol. 2008, 2007.

[54] E. Candes and T. Tao, "Near-optimal signal recovery from random projections: Universal encoding strategies?" Information Theory, IEEE Transactions on, vol. 52, no. 12, pp. 5406-5425, Dec 2006.

[55] E. Candes and J. Romberg, "11-magic: Recovery of sparse signals via convex programming," 2005

[56] S. Foucart and H. Rauhut, A mathematical introduction to compressive sensing. Springer, 2013, vol. 1, no. 3

[57] C. $\mathrm{Li}$, "An efficient algorithm for total variation regularization with applications to the single pixel camera and compressive sensing," Ph.D. dissertation, Rice University, 2009. 
[58] S. Mallat, "A theory for multiresolution signal decomposition: the wavelet representation," Pattern Analysis and Machine Intelligence, IEEE Transactions on, vol. 11, no. 7, pp. 674-693, Jul 1989.

[59] I. Daubechies, Ten Lectures on Wavelets. Society for Industrial and Applied Mathematics, 1992.

[60] R. Keys, "Cubic convolution interpolation for digital image processing," Acoustics, Speech and Signal Processing, IEEE Transactions on, vol. 29, no. 6, pp. 1153-1160, Dec 1981.

[61] D. L. Gall and A. Tabatabai, "Sub-band coding of digital images using symmetric short kernel filters and arithmetic coding techniques," International Conferance on Acoustics, Speech, and Signal Processing, vol. 2, pp. 761-764, April 1988.

[62] A. Cohen, I. Daubechies, and J. C. Feauveau, "Biorthogonal bases of compactly supported wavelets," Communications on Pure and Applied Mathematics, vol. 45, no. 5, pp. 485-560, 1992.

[63] M. Keramidas, V. Josserand, C. A. Righini, C. Wenk, C. Faure, and J. L. Coll, "Intraoperative near-infrared image-guided surgery for peritoneal carcinomatosis in a preclinical experimental model," British Journal of Surgery, vol. 97, no. 5, pp. 737-743, 2010.

[64] S. C. Park, M. K. Park, and M. G. Kang, "Super-resolution image reconstruction: a technical overview," Signal Processing Magazine, IEEE, vol. 20, no. 3, pp. 21-36, 2003. 